\title{
Vorstandswahlen der Arbeitsgemeinschaften der DRG auf dem 97. Deutschen Röntgenkongress, 04. - 07. Mai 2016 in Leipzig
}

Auf dem diesjährigen Röntgenkongress finden für folgende Arbeitsgemeinschaften Vorstandswahlen statt:

- DeGIR (Deutsche Gesellschaft für Interventionelle Radiologie)

- AG Gastrointestinal-/Abdominaldiagnostik

- AG Herzdiagnostik

- AG Informationstechnologie a@GIT

- AG Methodik \& Forschung (AGMF)

- AG Mammadiagnostik

- AG Physik und Technik (APT)

- AG Thoraxdiagnostik

Entsprechend der Arbeitsordnung der Arbeitsgemeinschaften bedürfen die zur Wahl vorgeschlagenen Kandidaten der Zustimmung des Vorstands der DRG (nihil obstat). Die Mitglieder der oben genannten Arbeitsgemeinschaften werden hiermit eingeladen, Kandidaten für den Vorstand der Wahlperiode 2016-2018 vorzuschlagen. Die Vorschläge müssen schriftlich eingereicht werden und sollten das Einverständnis der vorgeschlagenen Kandidaten enthalten.

\section{DeGIR (Deutsche Gesellschaft für Interventionelle Radiologie) $\nabla$}

Der Vorstand der DeGIR bittet seine Mitglieder um Kandidatenvorschläge für die Vorstandswahl 2016. Als Kandidaten können nur Mitglieder vorgeschlagen werden, die am 1. März 2016 ordentliche Mit- glieder der DeGIR waren. Vorschläge bedürfen der Schriftform und sind bis spätestens 12. März 2016 an den Vorstand der DeGIR zu Händen des Vorsitzenden, Prof. Dr. Arno Bücker, Homburg, oder an die Geschäftsstelle der DeGIR z. Hd. Frau Epsch zu senden.

\section{AG Gastrointestinal-|Abdominaldi- agnostik}

Der Vorstand der AG Gastrointestinal-/ Abdominaldiagnostik bittet seine Mitglieder um Kandidatenvorschläge für die Vorstandswahl 2016. Als Kandidaten können nur Mitglieder vorgeschlagen werden, die am 1. März 2016 ordentliche Mitglieder 
der Arbeitsgemeinschaft waren. Vorschläge bedürfen der Schriftform und sind bis spätestens 12. März 2016 an den Vorstand der Arbeitsgemeinschaft zu Händen der Vorsitzenden, Prof. Dr. Andreas Schreyer, Regenburg, oder an die Geschäftsstelle der DRG z. Hd. Frau Müller zu senden.

\section{AG Herzdiagnostik \\ $\nabla$}

Der Vorstand der AG Herzdiagnostik bittet seine Mitglieder um Kandidatenvorschläge für die Vorstandswahl 2016. Als Kandidaten können nur Mitglieder vorgeschlagen werden, die am 1. März 2016 ordentliche Mitglieder der Arbeitsgemeinschaft waren. Vorschläge bedürfen der Schriftform und sind bis spätestens 12. März 2016 an den Vorstand der Arbeitsgemeinschaft zu Händen des Vorsitzenden Prof. Dr. Matthias Gutberlet, Leipzig, oder an die Geschäftsstelle der DRG z. Hd. Herrn Völker zu senden.

\section{AG Informationstechnologie a@GIT}

Der Vorstand der AG Informationstechnologie a@GIT bittet seine Mitglieder um Kandidatenvorschläge für die Vorstandswahl 2016. Als Kandidaten können nur Mitglieder vorgeschlagen werden, die am 1. März 2016 ordentliche Mitglieder der Arbeitsgemeinschaft waren. Vorschläge bedürfen der Schriftform und sind bis spätestens 12. März 2016 an den Vorstand der Arbeitsgemeinschaft zu Händen des Vorsitzenden Prof. Dr. E. Kotter, Freiburg, oder an die Geschäftsstelle der DRG z. Hd. Herrn Völker zu senden.

\section{AG Methodik \& Forschung (AGMF)}

Der Vorstand der AG Methodik \& Forschung (AGMF) bittet seine Mitglieder um Kandidatenvorschläge für die Vorstandswahl 2016. Als Kandidaten können nur Mitglieder vorgeschlagen werden, die am 1. März 2016 ordentliche Mitglieder der Arbeitsgemeinschaft waren. Vorschläge bedürfen der Schriftform und sind bis spätestens 12. März 2016 an den Vorstand der Arbeitsgemeinschaft zu Händen des Vorsitzenden Dr. med. Michael Eisenblätter, Münster oder an die Geschäftsstelle der DRG z. Hd. Herrn Völker zu senden.

\section{AG Mammadiagnostik $\nabla$}

Der Vorstand der AG Mammadiagnostik bittet seine Mitglieder um Kandidatenvorschläge für die Vorstandswahl 2016. Als Kandidaten können nur Mitglieder vorgeschlagen werden, die am 1. März 2016 ordentliche Mitglieder der Arbeitsgemeinschaft waren. Vorschläge bedürfen der Schriftform und sind bis spätestens 12. März 2016 an den Vorstand der Arbeitsgemeinschaft zu Händen des Vorsitzenden Herrn Prof. Dr. Markus MüllerSchimpfle, Frankfurt oder an die Geschäftsstelle der DRG z. Hd. Frau Engelhardt zu senden.

\section{AG Physik und Technik (APT) $\nabla$}

Der Vorstand der AG Physik und Technik (APT) bittet seine Mitglieder um Kandidatenvorschläge für die Vorstandswahl 2016. Als Kandidaten können nur Mitglie- der vorgeschlagen werden, die am 1. März 2016 ordentliche Mitglieder der Arbeitsgemeinschaft waren. Vorschläge bedürfen der Schriftform und sind bis spätestens 12. März 2016 an den Vorstand der Arbeitsgemeinschaft zu Händen des Vorsitzenden Dr. Georg Stamm, Hannover, oder an die Geschäftsstelle der DRG z. Hd. Herrn Völker zu senden.

\section{AG Thoraxdiagnostik}

Der Vorstand der AG Thoraxdiagnostik bittet seine Mitglieder um Kandidatenvorschläge für die Vorstandswahl 2016. Als Kandidaten können nur Mitglieder vorgeschlagen werden, die am 1. März 2016 ordentliche Mitglieder der Arbeitsgemeinschaft waren. Vorschläge bedürfen der Schriftform und sind bis spätestens 12. März 2016 an den Vorstand der Arbeitsgemeinschaft zu Händen des Vorsitzenden Prof. Dr. C. P. Heußel, Heidelberg, oder an die Geschäftsstelle der DRG z. Hd. Frau Engelhardt zu senden. 